\title{
Continuous Assessment of Left Ventricular Volume by Ultrasonic Analogue Conversion System
}

\author{
Yasumi Uchida, M.D., Kiyoshi Inoue, M.D., Tadashi Korde, M.D., \\ Yutaka Takabatake, M.D., and Akihito Kato, M.D.
}

\begin{abstract}
SUMMARY
Applicability of ultrasonic analogue conversion system devised by us for assessments of left ventricular volume was examined in patients with and without heart disease. The conversion system could sample the echoes from the endocardial surfaces of the interventricular septum and the posterior left ventricular wall. The conversion system could also calculate automatically the minor axis and volume of the left ventricle. End-diastolic, end-systolic, and stroke volumes calculated by the conversion system were close to those calculated from biplane ventriculograms and to those calculated from photographic records of B-mode echo-display. Change in left ventricular volume caused by respiration ranged up to $17.5 \%$. Spontaneous variations in left ventricular volume during continuous monitoring was up to $7.5 \%$. End-diastolic, end-systolic, and stroke volumes were reduced by sublingual administration of nitroglycerin. The results indicate applicability of the ultrasonic analogue conversion system for continuous assessments of left ventricular volume in man.
\end{abstract}

\section{Additional Indexing Words:}

Fixing apparatus of echo-transducer Biplane ventriculography Respiratory change Reproducibility Nitroglycerin

7 HE ability to estimate left ventricular dimensions and function by echoI cardiography was established by Feigenbaum, Fortuin, Pombo, and others. ${ }^{31-7), 91,11)}$ The echoes from the left ventricle are usually photographed on fixed or running films. Although photographic method of recording enables movements of several tissues to be studied simultaneously, it is timeconsuming to assess the motion and dimension of a given structure quantitatively and instantaneously from the conventional photographic records. In order to avoid this shortcoming, Uchida et al devised a time-to-voltage analogue conversion system which can select 2 echoes simultaneously; one from the left ventricular surface of the interventricular septum and another from the endocardium of the posterior left ventricular wall. ${ }^{13}$ ) This system

From the Second Department of Internal Medicine, Faculty of Medicine, University of Tokyo, Hongo, Tokyo 113, Japan.

Received for publication March 26, 1976. 
also enables automatic and instantaneous calculation not only of the minor axis but also of the volume of the left ventricle. Although applicability of this system for measurement of left ventricular dimensions in anesthetized dogs has been demonstrated, ${ }^{13)}$ clinical applicability of this system is unclear. Therefore, applicability of this conversion system in patients with and without heart disease has been examined in this study.

\section{Materials And Methods}

1. Ultrasonic analogue conversion sytem

The refrector echo was presented in the form of an amplitude-modulated time-base on an A-scope. At the same time, the echo was displayed on a brightness modulated time-base, the B-scope.

Successive 2 gating signals were produced at $820 \mathrm{~Hz}$ and were displayed on the A-scope. The position and width of the gating signals on the A-scope were changed manually.

\section{Gate A:}

The first gate (gate A in Fig. 1) sampled the downstroke of the echo within it. When there were more than 2 echoes with a voltage above the sampling threshold voltage within the gate, the downstroke of the first echo was sampled. Sampling portion of the downstroke of the echo could be changed by changing the sampling threshold. Usually, the sampling threshold was adjusted to sample a portion close to the beginning of the downstroke. A square-wave sampling pulse 0.5 mic-

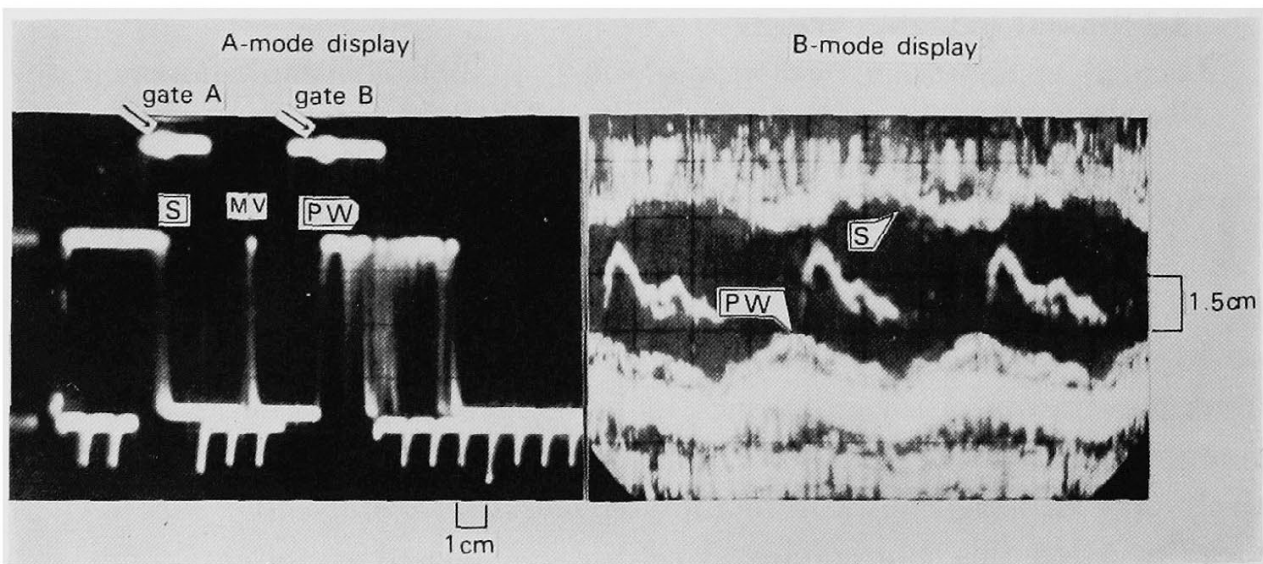

Fig. 1. Left half: A-mode display of the gate pulses (gate $\mathrm{A}$ and $\mathrm{B}$ ), sampling signals (arrows) and the echoes from the left ventricle in a patient without heart disease. FTC of the A-scope was not used to obtain the endocardial echoes sufficient for sampling by the conversion system. $\mathrm{S}=$ interventricular septum. $\mathrm{PW}=$ posterior left ventricular wall. $\mathrm{MV}=$ mitral valve.

Right half: B-mode display of the echoes from the left ventricle in the same patient. 
rosec in duration was produced at the sampled portion of the echo. A segment of the saw-teeth wave which was generated simultaneously with the gating pulses, was sampled during the sampling pulse was generated. ${ }^{13)}$ The sampled segment of the saw-teeth wave was maintained to the beginning of the next sampling pulse. The voltage of the sampled segment of the saw-teeth wave corresponded to the distance from the echo-transducer to the sampled portion of the refractor. The train of the sampled segments of the saw-teeth waves were smoothed by a filter of $15 \mathrm{~Hz}$ and were recorded. The sampling pulse was differentiated and clamped to provide a signal with brightness-modulated beam on the A-scope with a delay of 0.5 microsec to the sampled portion of the refractor echo (Fig, 1). Thus, it was possible to identify the sampled portion of the echo.

Gate B:

The second gate (gate B in Fig. 1) sampled the upstroke of the echo within it. When there were more than 2 echoes with sufficient voltage for sampling, the upstroke of the first echo was sampled. A sampling pulse was generated at the sampled portion of the echo. Usually, sampling threshold was adjusted to sample the beginning of the upstroke. Further process was the same as that in gate A (Fig. 1).

The voltage of the segment of the saw-teeth wave which was sampled by gate A was subtracted from that sampled by gate $B$. The output voltage after subtraction corresponded to the distance between the 2 sampled portions of the refractors. In this study, the echo from the left ventricular surface of the interventricular septum was sampled by gate $A$ and the echo from the endocardial surface of the posterior left ventricular wall was sampled by gate B. The output voltage after subtraction, therefore, corresponded to the minor axis of the left ventricle. Left ventricular volume was calculated automatically by a circuit of $\pi \mathrm{D}^{3} / 3$ ( $\mathrm{D}=$ minor axis) assuming the left veutricle as an ellipsoid according to Feigenbaum. ${ }^{3)}$

\section{Calibration:}

The position and width of the gates were obtained from the voltage which corresponded to the distances from the echo-transducer to the beginning and the end of the gating pulses and were recorded using multivibraters which produced squarewaves at $2 \mathrm{~Hz}$. At the same time, the position and width of the gates thus obtained were displayed digitally on the frontal panel of the converter. Stepwise pulses, each of which corresponded to $1 \mathrm{~cm}$, were also produced for calibration of the minor axis. The stepwise pulses were cubed by a circuit of $\pi \mathrm{D}^{3} / 3$ for calibration of volume. ${ }^{13}$ )

2. Fixing apparatus of echo-transducer

In this study, an apparatus which fixed the direction and position of the echotransducer was used. In this apparatus, the transducer was included in a plastic chamber and the angle of the transducer could be changed up to $35^{\circ}$ toward any direction and could be fixed. The plastic chamber was fixed on the chest wall with surgical tapes or belts (Fig. 2).

3. Comparison of left ventricular volume calculated by ultrasonic analogue conversion system to that calculated from biplane ventriculograms. 


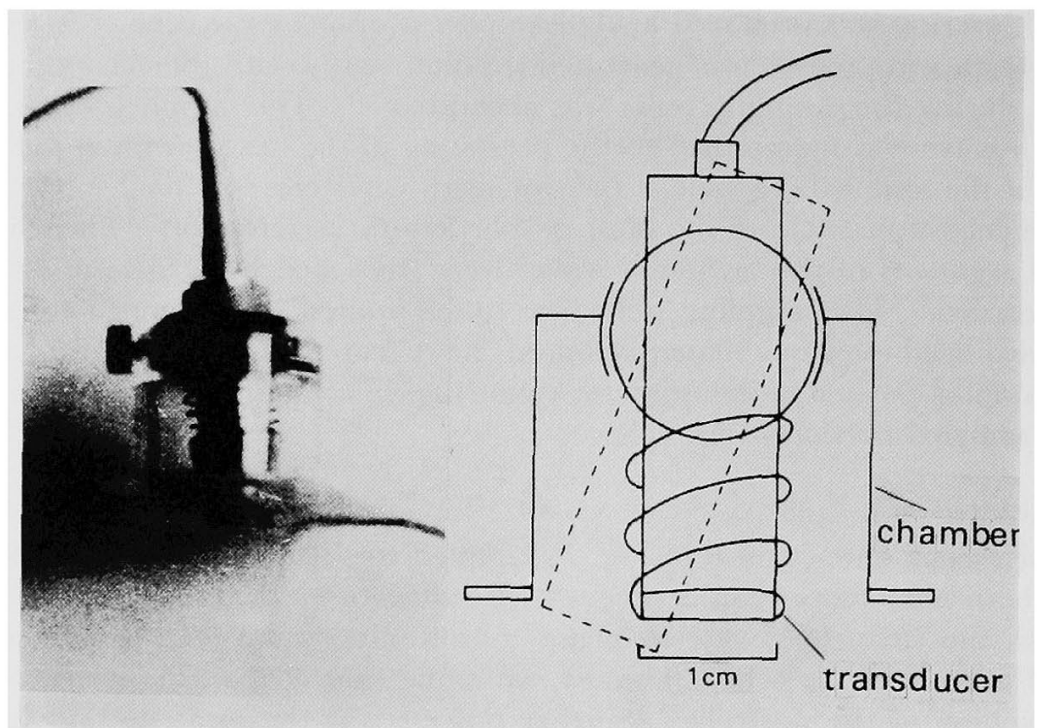

Fig. 2. Left half: a fixing apparatus of the echo-transducer. Right half: schematic representation of the fixing apparatus.

During left ventricular catheterization, the echo-transducer, $1 \mathrm{~cm}$ in diameter and with the fixing apparatus, was located over a water-soluble sound transmission gel interface at the fourth or fifth intercostal space adjacent to the left sternal border and angled posteriorly until a plane was found which included the interventricular septum and the endocardial surface of the posterior left ventricular wall just below the anterior leaflet of the mitral valve. The echoes from the left ventricle were displayed on the A- and B-scopes. In order to obtain the endocardial echoes sufficient for sampling by the conversion system, FTC of the A-scope was not used (Fig. 1). The position and width of the gates and the sampling threshold were adjusted to sample the echoes from the septal and posterior endocardial surfaces of the left ventricle and not to sample the echoes from any other tissues. Thus, motions of the interventricular septum and the posterior left ventricular wall and left ventricular volume calculated by the conversion system were recorded on a mingograph simultaneously with electrocardiogram, phonocardiogram, and aortic or left ventricular pressure in 14 patients ( 3 with mitral stenosis, 3 with combined valvular disease, 2 with aortic regurgitation, 2 with idiopathic cardiomyopathy, 2 with angina pectoris, and one with mitral regurgitation and ventricular septal defect). Thereafter, biplane left ventriculography was performed. Average value of 10 cardiac cycles of left ventricular volume calculated by the conversion system was compared to that calculated from the ventriculograms by the method of Dodge and Sandler. ${ }^{2)}$ In 8 of these patients, left ventricular volume calculated from the conventional photographic records of B-mode display using the method of Feigenbaum $^{3)}$ was compared to that calculated from ventriculograms. Photographic records and the records by the conversion system which were obtained after respiration was stopped at the early stage of expiration were used for calculation of the volume. In 8 patients who underwent and in 6 other patients (2 with idiopathic cardiomyopathy, 2 with mitral stenosis, 1 with hyperthyroidism, and 1 without heart 
disease), left ventricular volume calculated by the conversion system was compared to that calculated from the photographic records of B-mode display.

4. Effects of respiration on assessments of left ventricular volume by ultrasonic analogue conversion system

Respiratory variations in left ventricular volume were studied in 14 patients (6 with essential hypertension, 4 with combined valvular disease, 2 with angina pectoris, and 2 without heart disease). In these patients, the left brachial artery was cannulated to monitor the systemic blood pressure.

5. Continuous monitoring of left ventricular volume by ultrasonic analogue conversion system

Time-to-time variation in left ventricular volume during continuous recording for $20 \mathrm{~min}$ at rest was examined in 12 patients ( 8 with essential hypertension, 2 with angina pectoris, 1 with aortic regurgitation, and 1 with myocardial infarction). In these patients, the left brachial arterial pressure was monitored. Average value of 10 cardiac cycles which was obtained at the beginning of study was compared to those obtained at 5, 10, 15, and 20 min thereafter. The records during breath holding at the early expiratory phase were used for comparison.

6. Reproducibility of left ventricular volume measured by ultrasonic analogue conversion system

In 11 patients ( 4 with combined valvular disease, 2 with idiopathic cardiomyopathy, one with aortic stenosis, mitral stenosis, mitral regurgitation, and hyperthyroidism), the echo-transducer was located as described above and the left ventricular volume was calculated by the conversion system. The position of the fixing apparatus and the direction of the transducer were drawn on the chest wall by a skin-pencil and the transducer with apparatus was removed. Thereafter, the transducer 'with apparatus was placed at the same position. The same maneuver was repeated 4 times and changes in calculated left ventricular volume were examined.

7. Changes in left ventricular volume following sublingual administration of nitroglycerin

In 5 patients with essential hypertension and in 4 patients with angina pectoris, changes in left ventricular volume following sublingual administration of nitroglycerin $(0.6 \mathrm{mg})$ were examined. In these patients, the volume was recorded with electrocardiogram and the left brachial arterial pressure on a pen-recorder. The volume before was compared to that at $1,3,5,10,20$, and $25 \mathrm{~min}$ after the administration of the agent.

\section{Results}

1. Comparison of left ventricular volume calculated by ultrasonic analogue conversion system to that calculated from biplane ventriculograms

Fig. 1 shows motion of the interventricular septum and of the posterior left ventricular wall and the instantaneous changes in left ventricular volume 
in a patient without heart disease. An increase in volume due to atrial contraction was usually demonstrated in patients without heart disease (Fig. 3). The volume became maximum during the PQ segment or QRS of electrocardiogram and became minimum at or shortly later than the second heart sound (Fig. 3).

Comparison of the value of left ventricular volume calculated by the conversion system to that calculated from biplane ventriculograms was performed in patients with various heart diseases. The left ventricular enddiastolic volumes calculated by the conversion system were close to those calculated from the ventriculograms, correlation coefficient being 0.891 (Fig. 4). However, there was a tendency that the conversion system underestimated the volume in smaller left ventricles while overestimated it in larger left ventricles. The left ventricular end-systolic volumes were also close to those calculated from the ventriculograms, correlation coefficient being 0.814 (Fig. 5). The correlation in total stroke volume was, however, less close than in end-

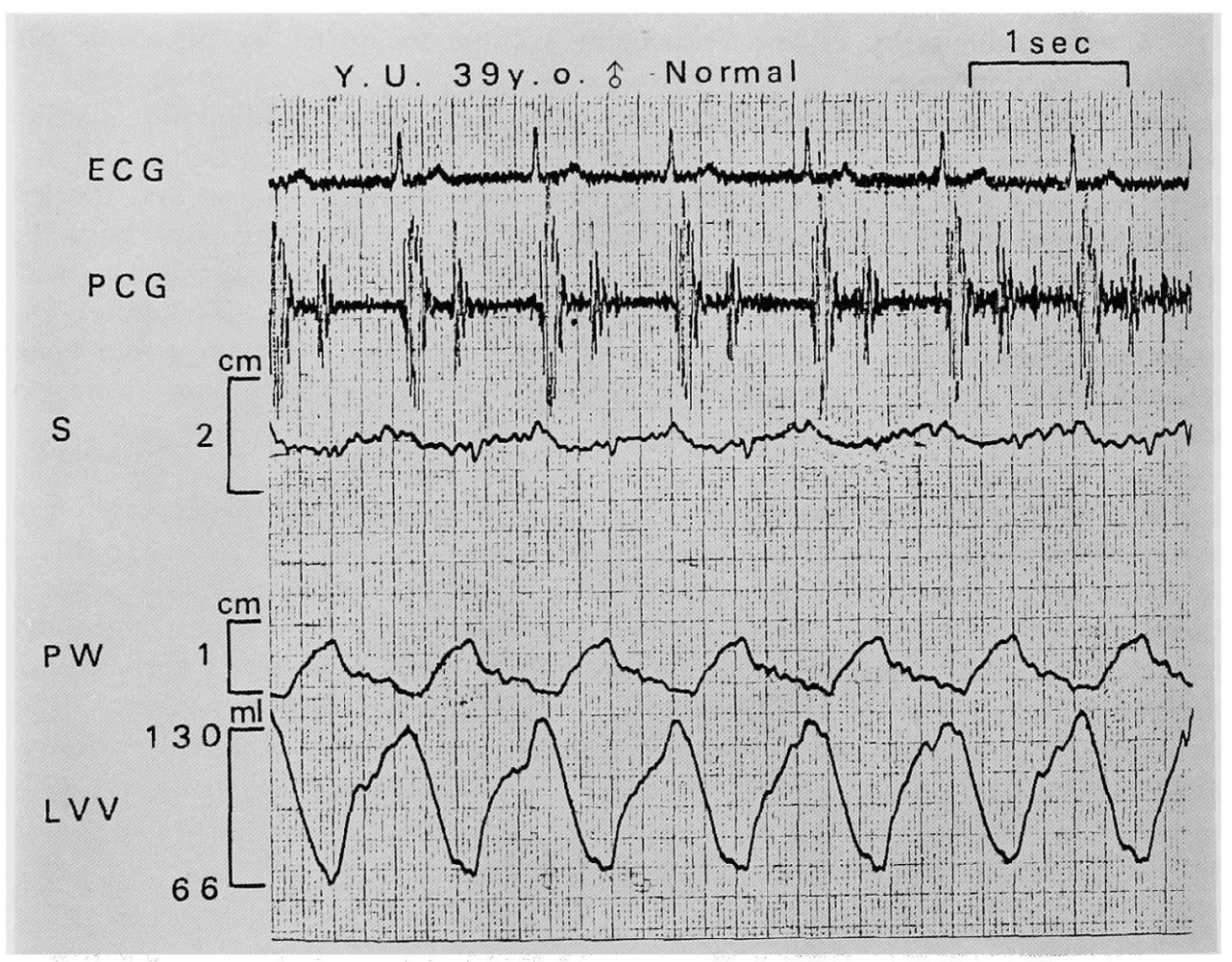

Fig. 3. From the top: electrocardiogram (ECG), phonocardiogram (PCG), motion of the interventricular septum (S), motion of the posterior left ventricular endocardium (PW), and left ventricular volume (LVV) in a patient without heart disease. 

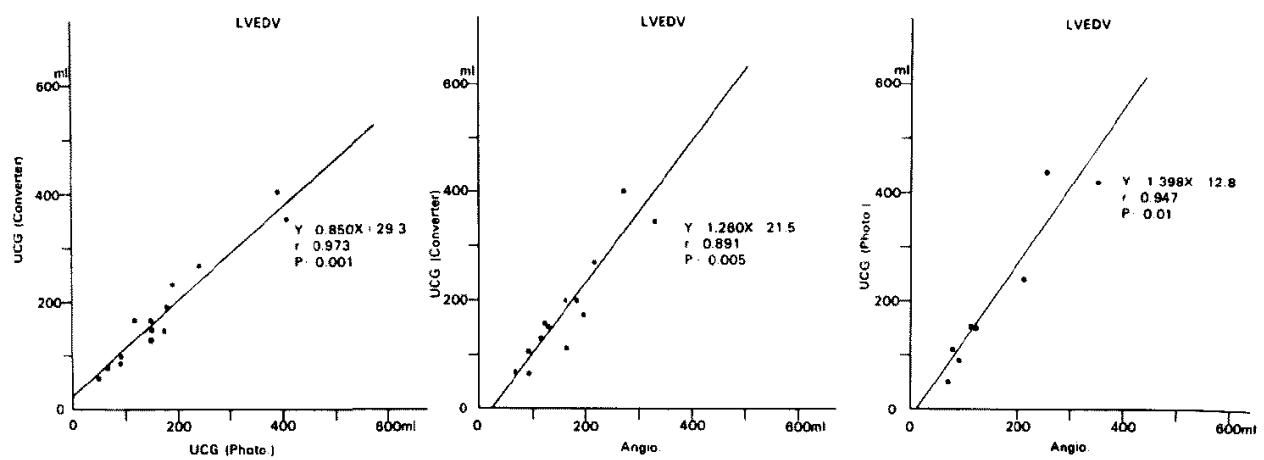

Fig. 4. Comparison of end-diastolic volume (LVEDV), end-systolic volume (LVESV), and total or net stroke volume (SV) calculated by the ultrasonic conversion system to those calculated from biplane ventriculograms. Converter $=$ ultrasonic conversion system. Angio=biplane ventriculography.
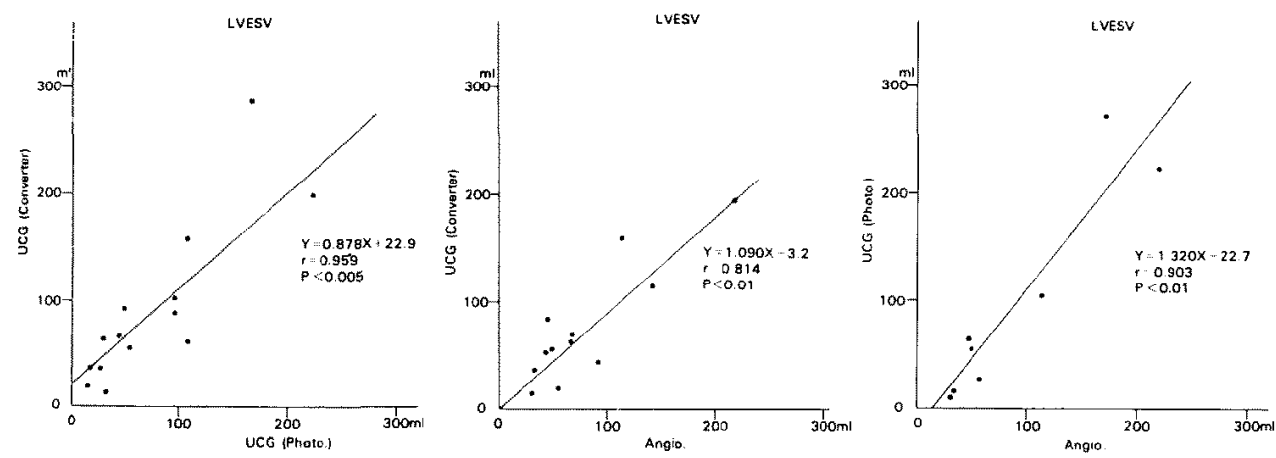

Fig. 5. Comparison of end-diastolic volume, end-systolic volume, and stroke volume calculated from the conventional photographic records of Bmode display to those calculated from biplane ventriculograms.
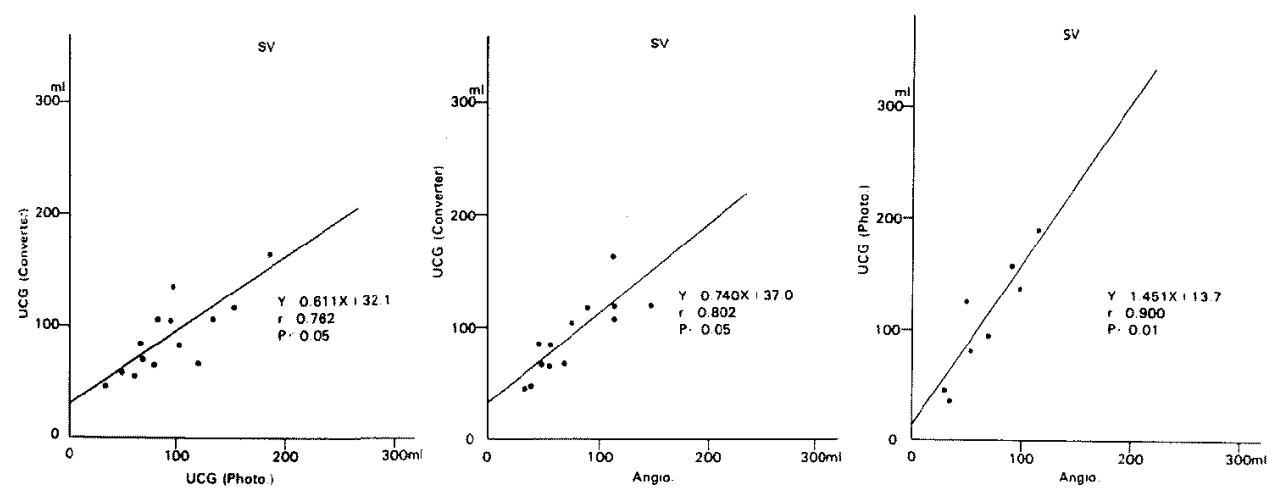

Fig. 6. Comparison of end-diastolic volume, end-systolic volume, and stroke volume calculated by the conversion system to those calculated from the photographic records of B-mode display. 
diastolic and end-systolic volumes (Fig. 6).

In 8 patients who underwent ventriculography, left ventricular volume calculated from the conventional photographic records was compared to that calculated from the ventriculograms. There were close correlations in enddiastolic, end-systolic and stroke volumes between these 2 methods. However, there was a tendency that photographic recording underestimated enddiastolic while overestimated stroke volume in smaller ventricles (Figs. 4, 5, and 6).

In 14 patients, left ventricular volume calculated by the conversion system was compared to that calculated from photographic records. Although there were close correlations in end-diastolic, end-systolic, and stroke volumes between these 2 methods, there was a tendency that the conversion system overestimated the volumes in smaller ventricles (Figs. 4, 5, and 6).

In 3 patients whose left ventricular volumes were not shown in Figs. 4, 5 , and 6 , left ventricular volume could not be calculated by the conversion system since the echo from the endocardial surface of the posterior left ventricular wall sufficient for sampling could not be obtained.

2. The effects of respiration on assessment of left ventricular volume by ultrasonic analogue conversion system

In 14 patients with various heart diseases, the effect of voluntary respiration on left ventricular volume was examined. Usually, end-diastolic volume became smaller while end-systolic volume became larger during inspiration. These changes were abolished by breath-holding at early expiration (Fig. 7

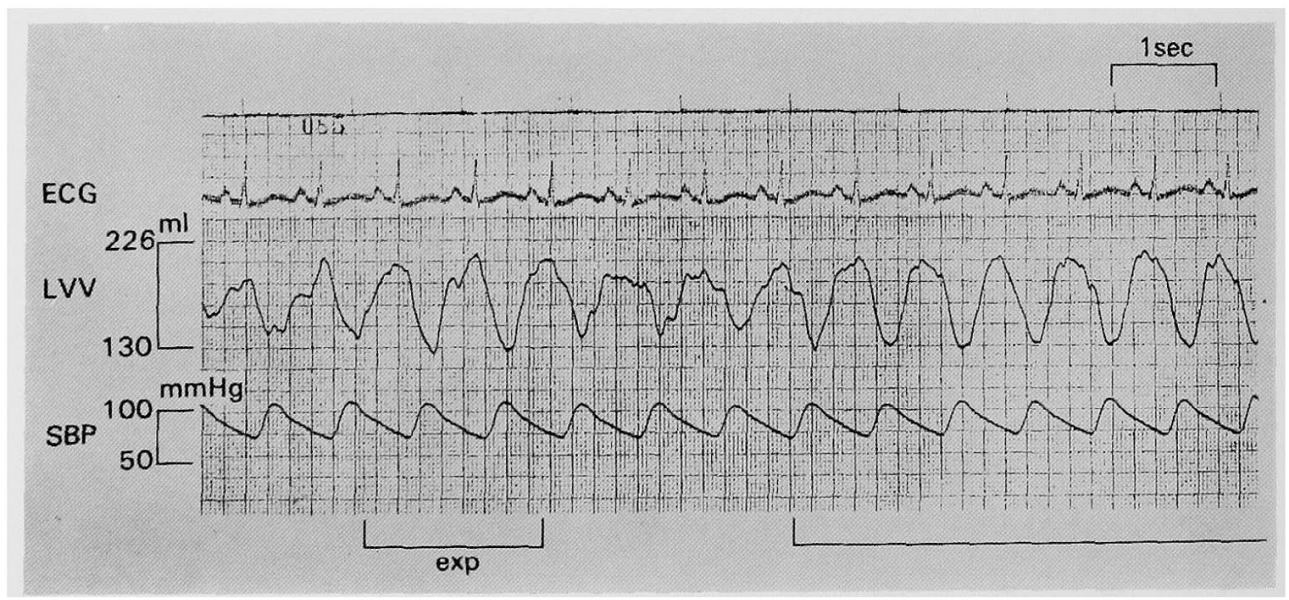

Fig. 7. Respiratory changes in left ventricular volume in a patient with angina pectoris. exp=expiration (the first horizontal bar). The second horizontal bar indicates stop of respiration at the early stage of expiratory phase. $\mathrm{SBP}=$ systemic blood pressure. 
and Table I). Average percent changes in end-diastolic, end-systolic, and stroke volumes between inspiration and expiration were up to 11.5, 13.2, and 17.5 , respectively (Table $\mathbf{I}$ ). Therefore, volume values obtained on early expiratory breath-holding were used in the other series of examination.

Table I. Respiratory Changes in Left Ventricular Volume

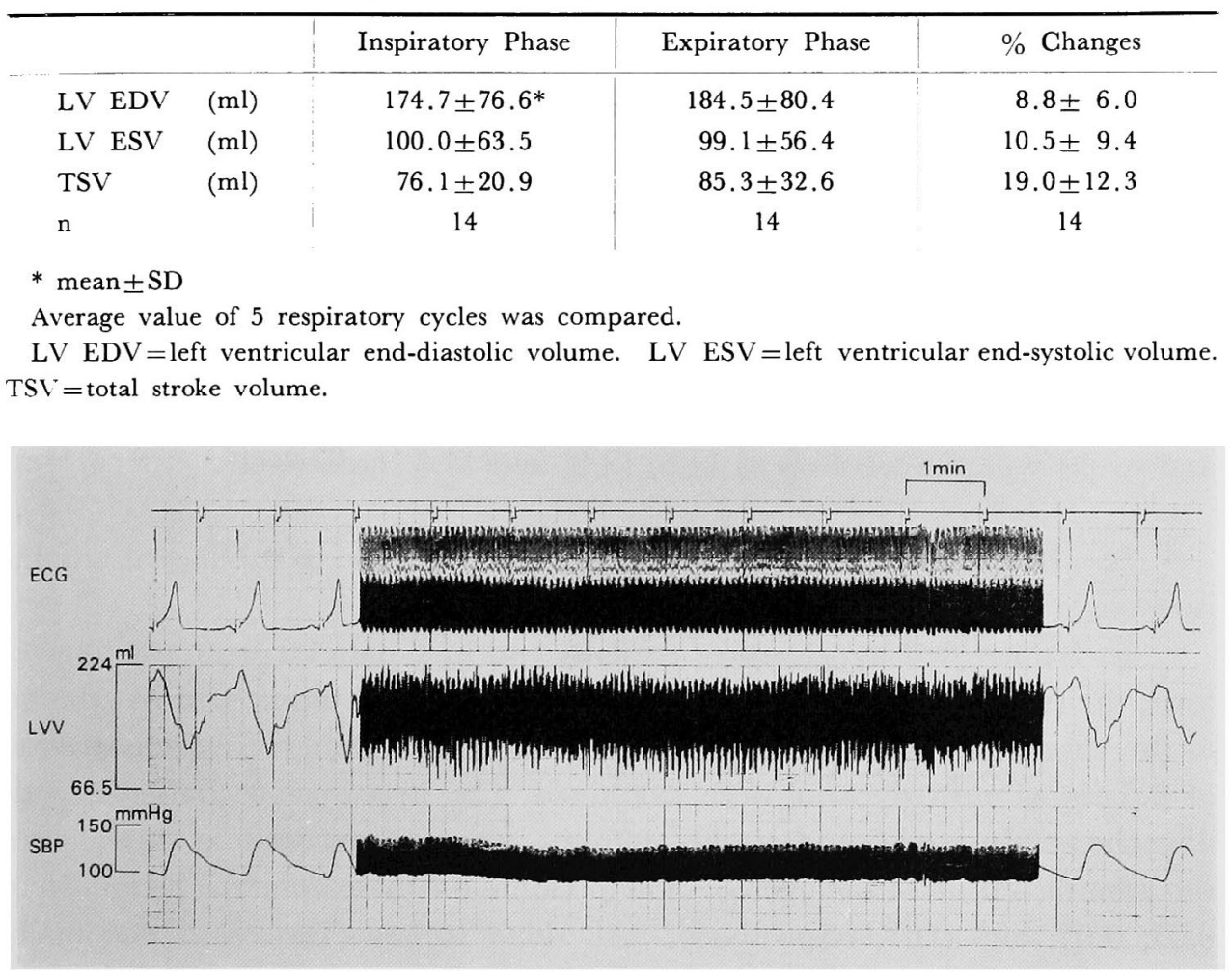

Fig. 8. A continuous record of left ventricular volume in a patient with essential hypertension.

Table II. Changes in Left Ventricular Volume during Continuous Monitoring by Ultrasonic Analogue Conversion System

\begin{tabular}{c|c|c|c|c|c}
\hline & Control & $5 \mathrm{~min}$ & $10 \mathrm{~min}$ & $15 \mathrm{~min}$ & $20 \mathrm{~min}$ \\
\hline LV EDV (ml) & $165.2 \pm 51.4$ & $166.5 \pm 51.5$ & $165.0 \pm 53.9$ & $164.0 \pm 51.4$ & $167.1 \pm 54.2$ \\
\% change & & $2.4 \pm 3.7$ & $2.5 \pm 3.8$ & $1.6 \pm 2.7$ & $1.3 \pm 2.1$ \\
LV ESV (ml) & $87.8 \pm 51.4$ & $85.9 \pm 49.9$ & $87.0 \pm 50.1$ & $86.1 \pm 49.7$ & $87.2 \pm 49.2$ \\
\% change & & $4.0 \pm 3.6$ & $4.2 \pm 4.5$ & $3.7 \pm 5.8$ & $5.0 \pm 6.8$ \\
TSV (ml) & $85.9 \pm 47.4$ & $81.6 \pm 33.8$ & $78.8 \pm 30.8$ & $80.1 \pm 28.5$ & $80.6 \pm 30.0$ \\
$\%$ change & & $7.5 \pm 12.5$ & $6.6 \pm 12.7$ & $6.9 \pm 10.0$ & $7.5 \pm 10.1$ \\
n & 11 & 11 & 11 & 11 & 11
\end{tabular}

Average value of 10 cardiac cycles which was obtained after respiration was stopped at the early stage of expiratory phase, was compared. 
3. Spontaneous changes in left ventricular volume calculated by ultrasonic analogue conversion system

In 12 patients, the change in left ventricular volume during supine rest was examined by continuous recording by the conversion system. The enddiastolic, end-systolic, and stroke volumes were altered along with, or without the changes in systemic blood pressure (Fig. 8). Maximum average percent changes in end-diastolic, end-systolic, and stroke volumes at various time intervals were $2.5,5.0$, and 7.5 , respectively (Table II).

4. Reproducibility of left ventricular volume measured by ultrasonic analogue conversion system

In 11 patients, the transducer with the fixing apparatus was replaced 4 times at the same position on the chest wall, and the left ventricular volume values calculated on each placement by the conversion system were compared to the initial ones. Average percent differences in end-diastolic, end-systolic, and stroke volumes were up to $11.5,13.2$, and 17.6 respectively.

5. The changes in left ventricular volume following sublingual administration of nitroglycerin

In 5 untreated hypertensive patients and in 4 patients with angina pectoris, the time course of the changes in left ventricular volume and its relation to the changes in systemic blood pressure following sublingual administration of $0.6 \mathrm{mg}$ of nitroglycerin were examined. In hypertensive patients, enddiastolic, end-systolic, and stroke volumes began to decrease at 5 min after the administration of the agent. The $\mathrm{p}$ values for these volume changes were $0.05,0.05$, and 0.01 , respectively. All these volumes decreased maximally between 10 and $15 \mathrm{~min}$ after the administration and returned toward the control values by $25 \mathrm{~min}$ (Table IV). Although both systolic and diastolic blood pressures fell 5-10 min after the drug administration, these falls were not significant (Table IV).

Table III. Reproducibility of Assessments of Left Ventricular Volume

\begin{tabular}{|c|c|c|c|c|c|}
\hline & 1st & & 2nd & $3 \mathrm{rd}$ & 4 th trial \\
\hline LV EDV $\quad(\mathrm{ml})$ & $237.8 \pm 136.1$ & & $226.6 \pm 124.8$ & $239.0 \pm 138.6$ & $242.8 \pm 150.5$ \\
\hline$\%$ difference & & & $8 . \mathrm{i} \pm 5.2$ & $11.5 \pm 9.5$ & $9.7 \pm 5.1$ \\
\hline LV ESV $\quad(\mathrm{ml})$ & $109.3 \pm 80.3$ & & $102.6 \pm 68.6$ & $104.0 \pm 77.8$ & $102.0 \pm 73.6$ \\
\hline$\%$ difference & & & $10.2 \pm 8.2$ & $13.2 \pm 11.5$ & $12.8 \pm 10.3$ \\
\hline TSV $\quad(\mathrm{ml})$ & $128.4 \pm 61.5$ & i & $124.0 \pm 67.9$ & $134.9 \pm 72.2$ & $140.3 \pm 82.4$ \\
\hline$\%$ difference & & & $12.6 \pm 13.6$ & $17.5 \pm 13.9$ & $17.6 \pm 14.8$ \\
\hline $\mathrm{n}$ & 11 & & 11 & 11 & 11 \\
\hline
\end{tabular}




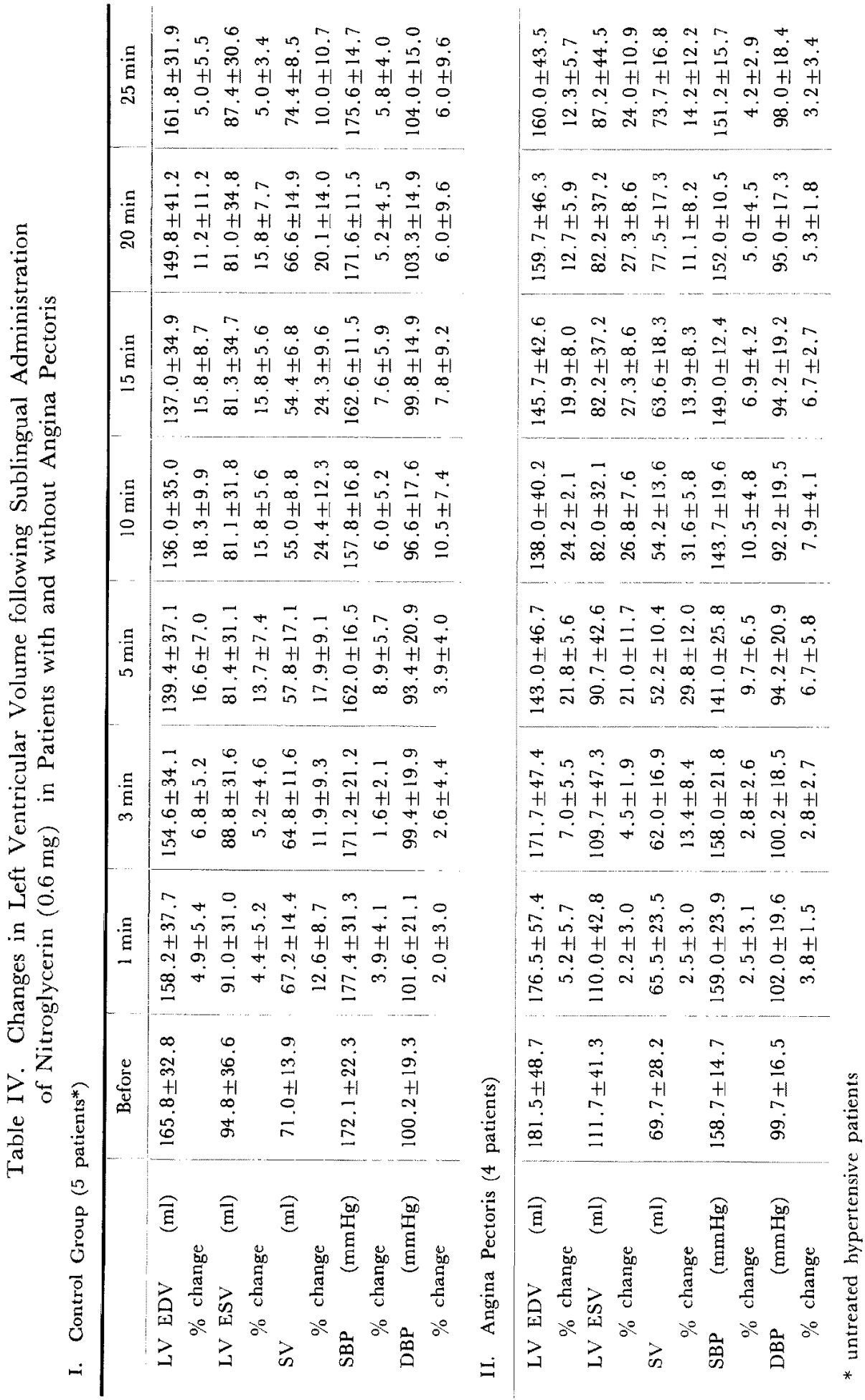




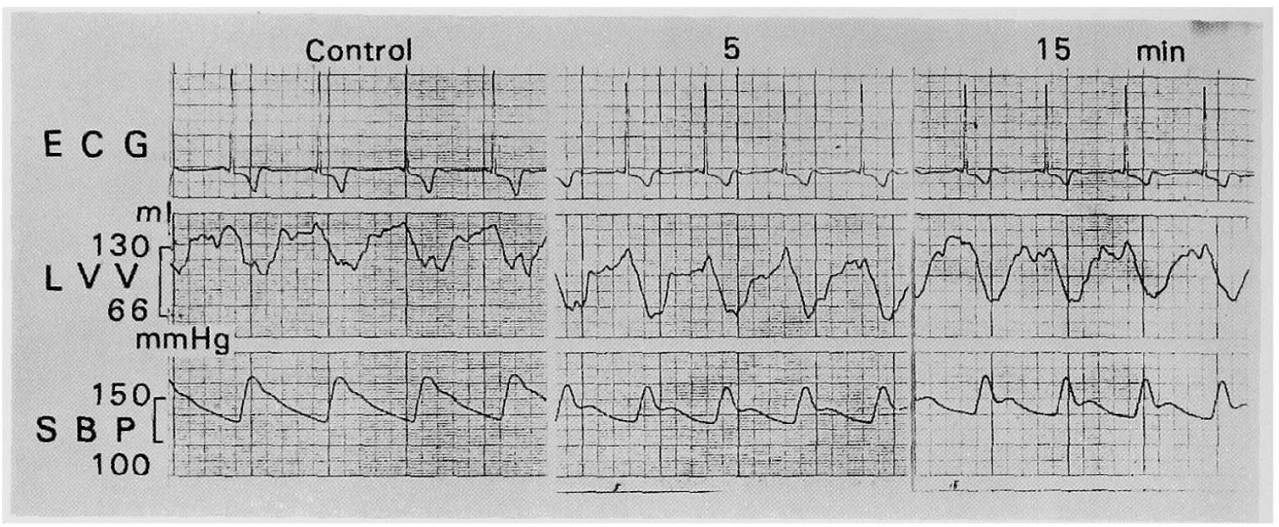

Fig. 9. The changes in left ventricular volume following sublingual administration of nitroglycerin $(0.6 \mathrm{mg})$ in a patient with angina pectoris.

In patients with angina pectoris, end-diastolic, end-systolic, and stoke volumes began to decrease at $5 \mathrm{~min}$ after the drug administration as in the case of hypertensive patients. The $\mathrm{p}$ values for these volume changes were $0.01,0.01$, and 0.01 , respectively. The decrease in these volumes became maximum at $10 \mathrm{~min}$ after the administration and gradually returned toward the control values. Although both systolic and diastolic blood pressures fell after the administration of nitroglycerin, the changes were not significant as in the case of hypertensive patients (Fig. 9 and Table I)

\section{Discussion}

In this study, the left ventricular end-diastolic, end-systolic, and stroke volumes calculated by the ultrasonic analogue conversion system using the formula of $\pi \mathrm{D}^{3} / 3$ ( $\mathrm{D}=$ minor axis), assuming the left ventricle as an ellipsoid of revolution, ${ }^{3)-5)}$ were close to those calculated from biplane ventriculograms in patients with various heart diseases. Feigenbaum et al examined the relationship between the left ventricular volume calculated from the photographic records of B-mode echo-display and that calculated from biplane ventriculograms and observed a close correlation $(r=0.895)$ between them. ${ }^{51}$ Kusukawa et al also examined the left ventricular volume calculated from the photographic records of B-mode display and from cineventriculograms in various heart diseases and obtained correlation coefficients ranging from 0.75 to $0.90 .{ }^{8}$ ) The correlation coefficients obtained in this study were close to these published values.

Kusukawa et al noted that photographic recording of B-mode display underestimates the volume in small chambered left ventricle while overestimates it in large chambered left ventricle. ${ }^{8)}$ The same tendency was ob- 
served in this study. Pombo et al noted that echocardiography is not an accurate method for volume estimation in patients with left ventricular dyssynergy. ${ }^{10}$ Since volume estimation in patients with obvious left ventricular dyssynergy was not performed in this study, it remains unclear whether the conversion system is applicable for these patients or not.

Voluntary respiration caused a change up to $17.5 \%$ in left ventricular volume. Changes in hemodynamic state, thoracic wall motion, and positional change of the heart due to respiration may have caused the variation in volume estimate. Since the left ventricular volume remained stable for several seconds after respiration was stopped at the early expiratory phase, the volume obtained during this period was used for comparison in the other examination,

During continuous monitoring by the conversion system at supine rest, the change in left ventricular volume was minimal. This minimal change can be attributed to circulatory changes or a small diplacement of the echotransducer. Replacing the echo-transducer to the same position on the chest wall caused a difference up to $17.5 \%$ in volume estimate. The main cause of this difference may have been the change in sampling site of the endocardial surfaces.

A reduction in left ventricular end-diastolic, end-systolic, and stroke volumes following sublingual administration of nitroglycerin has been reported by several workers. ${ }^{1,121,14}$ In this study, significant decreases in these volumes following sublingual administration of the agent were observed in patients with angina pectoris and in those with essential hypertension. These changes occurred about $5 \mathrm{~min}$ after the administration. The finding is similar to that by Redwood et al.12) The changes in the volumes following nitroglycerin were much larger than those observed during continuous monitoring for $20 \mathrm{~min}$ without any intervention.

In this study, the left ventricular volume could not be calculated by the conversion system in a small number of patients since the echo from the endocardial surface of the posterior left ventricular wall sufficient for sampling could not be obtained. In these patients, the echo from the endocardial surface could be identified by the conventional photographic recording. This is a disadvantage of the ultasonic analogue conversion system. It was necessary to adjust the position and width of the gates manually to avoid sampling the echoes from the chordae and mitral valve. Such maneuver is not required for photographic recording, and this is another disadvantage of the conversion system.

Despite of these minor drawbacks, the simplicity of continuous volume monitoring by the ultrasonic analogue conversion system offers a merit for 
clinical use. The stability, reproducibility, and sensitivity of the method, shown in this study, indicate an applicability of this method for assessment of left ventricular volume in man.

\section{Acknowledgements}

The authors acknowledge Dr. Iwao Ito, Dr. Yasuro Sugishita, Dr. Katsuhiko Ozeki, Dr. Katsuhiko Nakajima, and Dr. Masaaki Kakihana for their cooperation during left heart catheterization.

\section{REFERENCES}

1. Brachfeld N, Bozer J, Gorlin R: Action of nitroglycerin on coronary circulation in normal and in mild cardiac subjects. Circulation 29: 697, 1959

2. Dodge HT, Sandler H, Baxlay WA et al: Use of biplane angiography for measurement of left ventricular volume in man. Am Heart J 60: 762, 1960.

3. Fejgenbaum H, Zaky A, Nasser WK: Use of ultrasound to measure left ventricular volume. Circulation 35: 1092, 1967

4. Feigenbaum $\mathrm{H}$, Wolfe SB, Popp $\mathrm{R}$ et al: Correlation of ultrasound with angiography in measurement of left ventricular diastolic volume. Am J Cardiol 23: 111, 1969

5. Feigenbaum H, Popp RL, Wolfe SB et al: Ultrasound measurements of the left ventricle: a correlative study with angiocardiography. Arch Int Med 129: 461, 1972

6. Fortuin NJ, Hood WP Jr, Sherman ME et al: Determination of left ventricular volume by ultrasound. Circulation 44: 575, 1971

7. Fortuin NJ, Hood WP Jr, Craige E, et al: Evaluation of left ventricular function by echocardiography. Circulation 46:26, 1972

8. Kusukawa R, Mashiro I, Tomonaga T: Comparison of left ventricular function measured by echocardiography to that measured by ventriculography. Naika 36: 774, 1975 (in Japanese)

9. Pombo JF, Troy BL, Russel RO Jr: Left ventricular volume and ejection fraction by echocardiography. Circulation 43: 493, 1971

10. Pombo JF, Russel RO, Rackley CE et al: Comparison of stroke volume and cardiac output determination by ultrasound and dye dilution in acute myocardial infarction. Am J Cardiol 27: 630, 1971

11. Popp RL, Wolfe SB, Hirata $\mathrm{T}$ et al: Estimation of right and left ventricular size by ultrasound. Am J Cardiol 24: 523, 1969

12. Redwood DR, Henry WL, Epstein SE: Evaluation of the ability of echocardiography to measure acute alteration in left ventricular volume. Circulation 50: 901, 1974

13. Uchida $\mathrm{Y}$, Inoue $\mathrm{K}$, Yoshimoto N: Instantaneous assessments of left ventricular dimensions and volume by ultrasonic analogue conversion system. I. An experimental study. Jap Heart $J$ (to be published)

14. Williams JF, Glick G, Braunwald E: Studies on cardiac dimensions in intact unanesthetized man. Circulation 32: 767, 1965 